häufig in Gesellschaft von Nephrodium Robertianum, diversen Asplenien und zahlreichen alpinen Felsptlanzen (Saxifraga aizoon, Hostii, tenella, stellaris, Primula auricula, Phyteuma Sieberi, Potentilla caulescens usw.) und die Zikade zirpt, wie im Süden, unermüdlich in der Krone der Mannaesche (Fraxinus ornus).

D. Im Norden wird das Flitscher Becken vom $2206 \mathrm{~m}$ hoben Rombonrücken und von den sogenannten Bretterwänden begrenzt, die voneinander durch das schmale, aber tiefe Tal des KoritnicaBaches getrennt werden.

Der Rombon ist auf seiner Südseite bis zu den stark verkarsteten, höhlenreichen Felspartien ober $1500 \mathrm{~m}$ mit Latschen, gegen Osten und Norden mit Rotbuchenwald bestanden, der bier gleichfalls fast bis zur Baumgrenze (für die Latsche!) aufsteigt. Ober $1500 \mathrm{~m}$ beginnen üppige Matten mit den häufigeren Mattenblumen (vergl. I, S. 193); auffällig ist nur das massenhafte Auftreten von Knautic longifolia, Dianthus Sternbergii und Gentiana anisodonta. Diese Matten gehen höher hinauf in edelweißreiche Triften über, deren Flora völlig jener des Wischberges gleicht. Sedum roseum ist hier ungemein häufig. Die schroffen $A b-$ hänge der Čukla und ihrer Nachbarschaft sind bis auf kleine Wäldchen von Buchen auf den vorspringenden Schultern der Rombonflanke unbewaldet, ja fast ganz vegetationslos, und bieten ein um so trostloseres Aussehen, wenn man aus dem üppigen 'Talkessel zu ihnen aufsteigt.

Der Rombon gehört, seiner Hochgebirgsflora nach, ganz dem julischen Systeme an und gleicht hierin der Wischberggruppe und den Salmspitzen. Auch das enge Tal des Predil-Koritnica-Baches ist diesem Florenbezirke zuzuzählen vom Predilpaß bis zur Flitscher Klause. Schon an der Straße können wir, und dies ist hier sehr bequem, die alpine Triftformation, das Latschengehölze, die alpine Felsheide, gegen Breth hin die alpinen Matten (beim Stollen in Unterbreth ist Lilium carniolicum in Gesellschaft von L. bulbiferum und martagon ziemlich häufig) und an den Abhängen um Breth den alpinen Mischwald studieren.

\title{
Beiträge zur Kenntnis der Flora von Süddalmatien.
}

Von Dr. Friedrich Morton (Wien).

(Mit 3 Textabbildungen.)

Ims nachfolgenden gebe ich eine Reihe von Pflanzenfunden bekannt, die von mir gelegentlieh pflanzengeographischer Arbeiten im südlichen Dalmatien gemacht wurden. 
Polypodium vulgare L. var. serratum Willd. Insel Curzola: auf Kalkfelsen in der Formation der Garrigue zwischen Curzola und Lombarda. Asplenium acutum Bory. Am Südabfall des Koziak bei Spalato in der Macchie oberhalb Sućurac.

Quercus coccifera L. Halbinsel Sabbioncello: am Südabfall des Monte Vipera vom Meere bis gegen $300 \mathrm{~m}$ als Macchienelement. Stellenweise noch in mächtigen, baumförmigen Exemplaren.

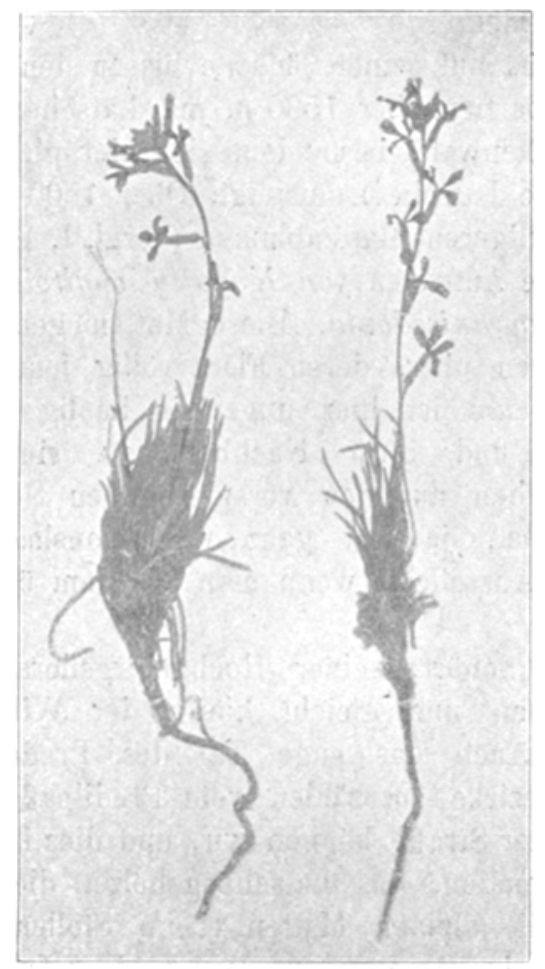

Abb, 1.

Ranunculus calthaefolius (Rchb.) Bl. N. Sch. Halbinsel Sabbioncello: in der Formation der steinigen Trift auf dem Monte Vipera.

Cardamine maritima Portenschl. Halbinsel Sabbioncello: an Steinmauern oberhalb Orebic.

Hesperis glutinosa Vis. Im Kalkgeröll am Fuße der Steilwände des Koziak bei Spalato; in der Formation der steinigen Triften auf dem Monte Vipera.

Matthiola tristis L, R. Br. var. italica Conti, subv. typica Conti. Halbinsel Sabbioncello: in der Formation der steinigen Triften am Sud. 
abfall 'des Monte Vipera., - Matthiola tristis erscheint als eine sehr formenreiche Pflanze, die nach Conti. (Classification et distribution des espèces européennies du genre Matthiola in "Bulletin .de L'Herbier Boissier", 'lome V, 1897, p. 31-59) in fünf Unterarten zerfält,. die den Verbreitungsbezirken; der Art entsprechen. (Alpiner, iberisch-provençaler, italisch-dalmatinischer, sizilischer und Balkanbezirk.) Die Pfanze des italisch-dalmatinischen Bezirkes. gehört zur var. italica Conti, die durch graugrüne Färbung, einfachen, nicht verzweigten, blattlosen Stamm and durch lineale, in Rosetten angeordnete Blätler gegenüber den übrigen Unterarten. gut gekennzeichnet erseheint. (Abb. 1.)..Von Conti wird sie innerbalb unserer Monarchie für Trebinje angegeben.

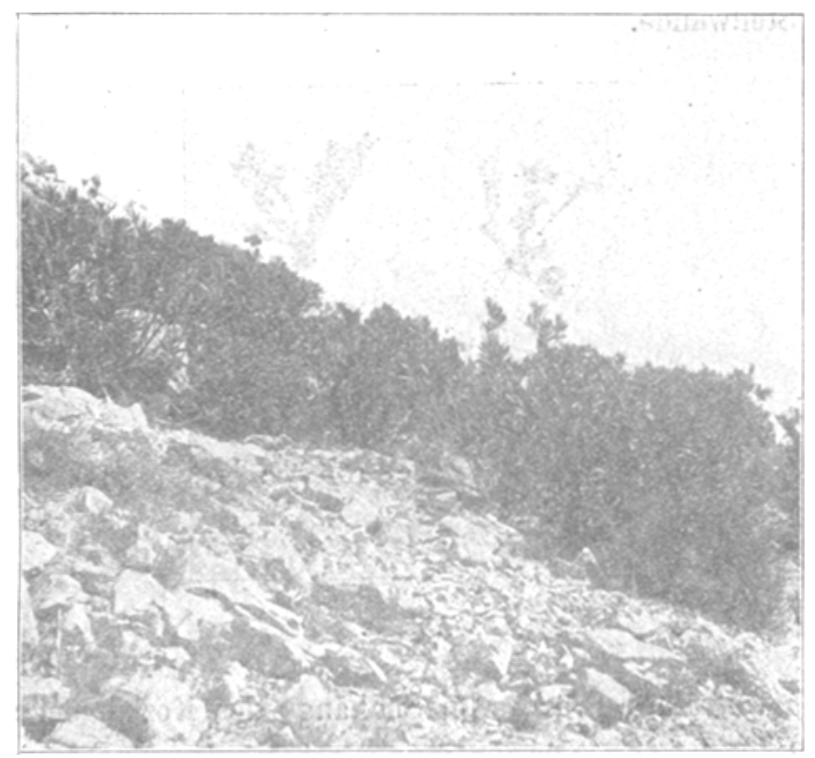

Abb. 2.

: Vesicaria graeca : Reut. Häufig am Fuße per Steilwände des Koziak bei Spalato und auf Schutthalden :am :SO-Abfall des Mónte Vipera.

Anthyllis Spruneri Boiss. Insel Curzola: in der Formation der Garrigue zwischen Curzola und Lombarda.

Nerium oleander L. Hallbinsél Sabbioncello: oberhalb des Dorfes Lampalovo am Südhange des Monte Vipera bildet Nerium ca. zwischen 200 und $300 \mathrm{~m}$ eine üppige Garrigue und ist mit Quercus coccifera, Euphorbia spinosa, Laurus nobilis, Coronilla emeroides u. a. vergesellschaftet. (Abb. 2.) 
Hyoseris scabra L. ${ }^{1}$ ) Insel Curzola: in der Formation der Garrigue und auf Ruderalplätzen zwischen Curzola und Lombarda. Auf einem Kalkfelsen fanden sich Zwergexemplare mit nur einer Blüte und nur 14 mm Höhe! (Abb. 3.)

Fritillaria tenella M. B. Insel Curzola: in der Formation der Garrigue zwischen Curzola und Lombarda.

Hyacinthus dalmaticus Backer. Halbinsel Sabbioncello: in großen Mengen am Südhang des Monte Vipera in einer kurzgrasigen Mulde bei ca. $750 \mathrm{~m}$.

Narcissus angustifolius Curt. Halbinsel Sabbioncello: in der Formation der steinigen Triften am Südhange des Monte Vipera.

Carex distachya Desf. Am Südhange des Koziak bei Spalato, am Fuße der Steilwände.

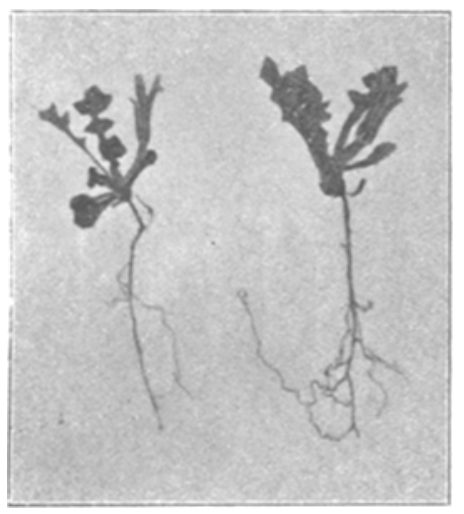

Abb. 3.

Avena convoluta Presl. Am Süuhhange des Koziak bei der Kirche Sv. Gospoja.

Sesleria tenuifolia Schrad. ${ }^{2}$ ) Halbinsel Sabbioncello: in der Formation der steinigen Triften am Südhang des Monte Vipera.

Orchis provincialis ${ }^{\mathbf{3}}$ ) Balb. In den Macchien oberhalb Suéurac am Südhange des Koziak; auf Schutthalden am S0-Abhange des Monte Vipera bis $650 \mathrm{~m}$.

Orchis quadripunctata $\mathrm{Cyr}^{3}$ ) In Ölgärten oberhalb Orebić.

Orchis Spitzelii Saut. ${ }^{3}$ ) In der Formation des mediterranen Schwarzföhrenwaldes am S0-Hange des Monte Vipera bis $800 \mathrm{~m}$.

1) revid. $A$. G in $z$ b e $r g$ er.

2) revid. E. $\mathrm{H}$ a $\mathrm{ck}$ el.

3) revid. H. Flei s ch mann. 